$R M x A C, \mathbf{5 3}, 169-173(2021)$

(c) 2021: Instituto de Astronomía, Universidad Nacional Autónoma de México

https://doi.org/10.22201/ia.14052059p.2021.53.35

\title{
AUTOMATING THE ALERT RESPONSE OF THE NU TRANSIENT TELESCOPE AT ASSY-TURGEN ASTROPHYSICAL OBSERVATORY (NUTTELA-TAO)
}

\author{
Z. Maksut ${ }^{1}$ and B. Grossan ${ }^{1,2}$
}

RESUMEN

Nuestro proyecto tiene como objetivo identificar la naturaleza física de los estallidos de rayos-gamma (GRB) a través de la medición de la distribución espectral en el óptico de esta emisión durante la fase de emisión gamma, que no suele pasar de 60 segundos. Estas mediciones requieren un telescopio óptico de rápido movimiento e instrumentación adecuada para responder de forma autónoma a las alertas GRB en tiempo real. El telescopio para transitorios de la Universidad de Nazarbayev en Assy-Turgen en el Observatorio Astrofísico (NUTTelATAO) tiene una apertura de $0.7 \mathrm{~m}$ y puede apuntar a cualquier lugar por encima del horizonte local en 8 segundos. Al dicho telescopio llegan las alertas GRB a través de internet por medio de la red de coordenadas de rayos-gamma. Y se mide la emisión óptica inmediata de cada GRB con el instrumento de observación simultánea en tres canales (BSTI), que incorpora 3 cámaras EMCCD, en las bandas Sloan g', r', i', permitiendo imágenes simultáneas de alta resolución temporal con exposiciones de unos pocos cientos de milisegundos por imagen. También describimos nuestro software automatizado para el sistema de control, incluyendo el algoritmo de control general, de control del telescopio, de control y actuación sistemas para el techo del recinto, de control del instrumento y entradas de clima y otros sensores. El sistema de software se basa en el lenguaje de datos GNU (GDL) en un entorno Linux, seleccionado para facilitar la escritura y software de eliminación de errores, permitiendo que los científicos del proyecto se familiaricen con el mismo pudiendo ser analizadas las imágenes prontamente. Se presentan los resultados obtenidos durante el período inicial de puesta en servicio.

\section{ABSTRACT}

Our project aims to identify the physical nature of gamma-ray burst (GRB) emission via measurement of the optical spectral shape of this emission during the prompt phase, usually lasting only $60 \mathrm{sec}$. These measurements require a fast-moving optical telescope and instrumentation to respond autonomously to real-time GRB alerts. The Nazarbayev University Transient Telescope at Assy-Turgen Astrophysical Observatory (NUTTelA-TAO) has a $0.7 \mathrm{~m}$ aperture, and can point anywhere above the local horizon in 8 seconds. We receive GRB Alerts via internet socket connection to the Gamma Coordinates Network (GCN) at the telescope site. We measure the GRB prompt optical emission with the Burst Simultaneous Three-Channel Imager (BSTI), which incorporates 3 EMCCD cameras, at Sloan g', r', and i' bands, for simultaneous high time-resolution imaging as fast as a few hundred millisecond per frame. We describe our automated control system software, including the overall control algorithm, control of the telescope, control and actuation systems for the enclosure roof, control of the instrument, and inputs from weather and other sensors. The software system is based on the GNU data language (GDL) in a Linux environment, selected for ease of writing and de-bugging software, familiarity to the project scientists, and image analysis capabilities. We give system performance results obtained during the early commissioning period.

Key Words: gamma-ray burst: general — instrumentation: miscellaneous — telescopes

\section{INTRODUCTION}

The scientific aim of our project is to measure the broad-band spectral shape of gamma-ray burst (GRB) prompt optical emission with high timeresolution. Only 3-8 prompt GRB detection per

\footnotetext{
${ }^{1}$ Energetic Cosmos Laboratory, Nazarbayev University, Kazakhstan.

${ }^{2}$ University of California, Berkeley Space Sciences Laboratory, USA.
}

year are expected from this experiment (Grossan et al. 2019), therefore, reliability is extremely important and an automation system with high reliability is required. We are using the Nazarbayev University Transient Telescope at Assy-Turgen Astrophysical Observatory (NUTTelA-TAO), a $0.7 \mathrm{~m}$ aperture modified Dall-Kirkham (CDK) commercially supplied telescope, with software modification, able to point to and track any target above the local horizon 
in $8 \mathrm{~s}$. The telescope is located at Assy-Turgen Astrophysical Observatory (ATO), near Almaty, Kazakhstan, at an altitude of $2750 \mathrm{~m}$, installed in October of 2018 ( $\mathrm{Li}$ et al. 2019). Temperatures of $-20 \mathrm{C}$ are common, and $-30 \mathrm{C}$ has been recorded; hardware must be selected appropriately. The principle instrument of the NUTTelA-TAO is the Burst Simultaneous Three-Channel Imager (BSTI) (Grossan et al. 2019), consisting of $31 \mathrm{Kx} 1 \mathrm{~K}$ EMCCD (electronmultiplied CCD) cameras in g', r' and i' Sloan filter bands, viewing the same field simultaneously via 2 dichroic beam splitters. We are also planning to develop Kilo array Microwave Kinetic Inductance detectors at the future next to BSTI to measure energy and quantity of arrival photon at the same time (Shafiee M. et al. 2019). Control of large telescope projects, with teams of engineers, may be designed from "the ground UP", building a unified power, hardware, and software control scheme. With our limited technical labor force and other resources, we had to control and communicate with a wide variety of off-the-shelf devices, instruments, and sensors, each with their own communication, programming, and control schemes. Another challenge of limited technical labor is our requirement to design a system that the scientists, not hardware or software professionals, can operate, maintain, and upgrade. In this paper we present our automation control solution, including hardware, software, communications and operation. We believe our unique approach holds lessons for similar efforts of limited resources, and scientist-driven requirements.

\section{HARDWARE}

Figure 1 shows the NUTTelA-TAO infrastructure: enclosure, satellite internet, weather station, and solar panels.

The block diagram of the hardware system is shown in Figure 2. The system has solar panels and batteries as its primary power source, and diesel generator as a backup power supply. The following subsections describe the hardware components of NUTTelA-TAO presented in Figure 2 in details.

\subsection{Computers, Automation Control box, and Ethernet}

Our system operates on computer control of devices based on alerts and sensor inputs. Due to the unprotected and unheated environment of the telescope enclosure building, we use industrial PCs (IPCs) rated to $-30 \mathrm{C}$ and generally suited to this environment. Our automation control box houses power supplies and relays which may be turned on

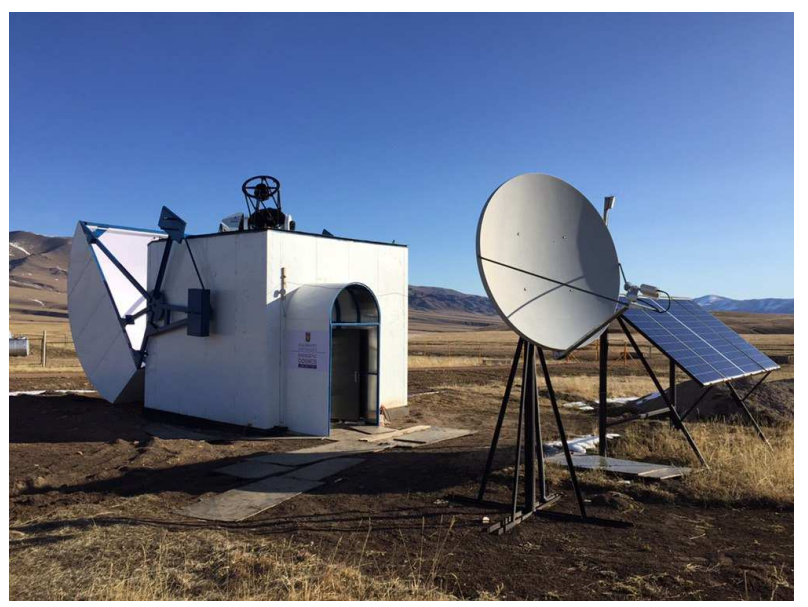

Fig. 1. Exterior View of the NUTTellA-TAO enclosure building and ancillary systems.

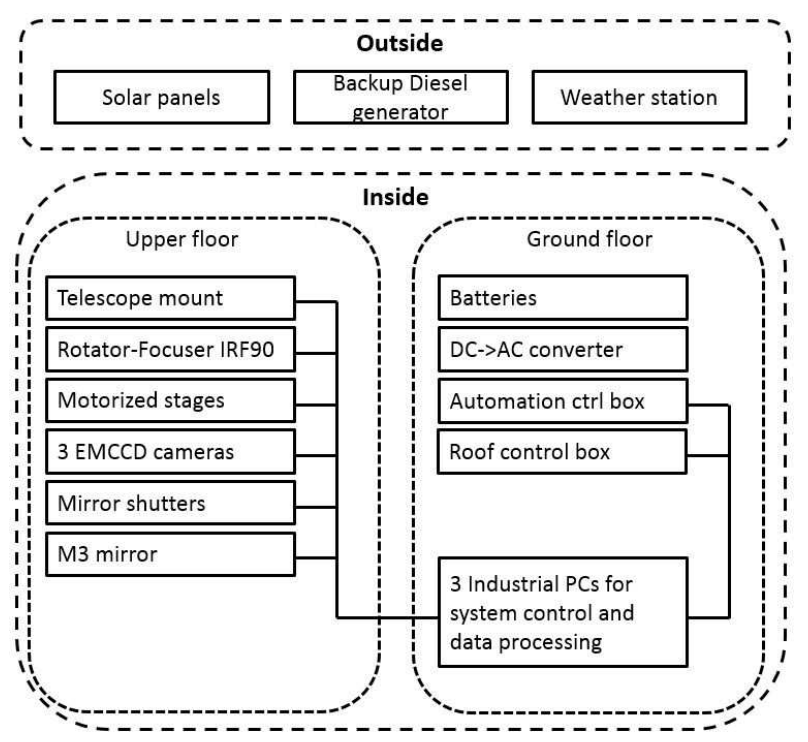

Fig. 2. Block diagram of hardware components.

and off via commands over Ethernet to the ioLogik E1212-T unit. This allows us to turn devices on-andoff with non-interactive computer control (it also allows us to do the same remotely). The box also contains a multi-port router with firewall that is connected to a satellite internet service. A LAN connects the computers to the ioLogik and other various devices of the system that are Ethernetcapable. Communication between automation control box and other equipment shown in Figure 3. Additionally, observation can be conducted remotely from the laboratory via secured internet connection. 


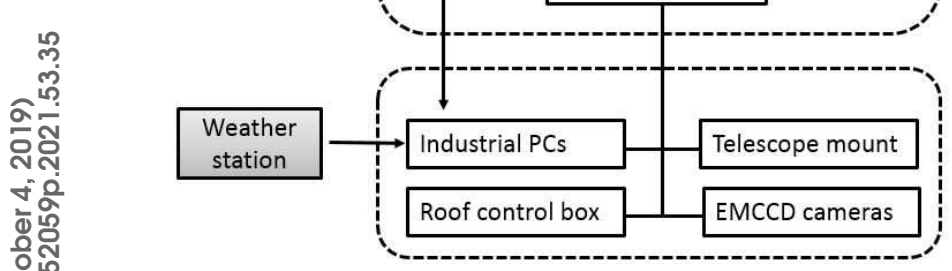

Fig. 3. Automation control box hardware components and connections.

\subsection{Weather and Environment Protection from Roof Control}

The observatory building (4 m x $4 \mathrm{~m}$ size) was built from a metal framework and covering panels. In order to keep the NUTTelA-TAO safe from weather damage (i.e. wind, dust, moisture, and precipitation) out telescope building includes a movable roof that seals the building from the exterior environment that may be opened and closed automatically (Figure 4). Outside the observatory building, 5 meters away we mounted a Boltwood Cloud Sensor II on a pole at a height of 6 meters. It has several sensors including sky temperature (an indication of clouds), wind speed, humidity level and dew point (Marchant et al. 2008). The "Captain" control program running on a Linux IPC monitors the weather information and decides if the roof should be open or closed. The roof moves (rotates) back to the North. In full-open position it blocks the view up to only 15 degrees above horizon. The telescope is mounted on an independent pillar, isolated from roof vibration. The roof control box has an SAJ 8000 programmable motor controller. This is connected to the ioLogik E1212-T, a "logic controller", a device that can turn relays on and off, allowing automated and remote control.

\subsection{Telescope and Sub-Systems}

The telescope has numerous independently controllable sub-systems; of most interest for our control here are the mirror covers ("shutters"), pointing, the IRF90, and the M3 mirror, which directs light toward the BSTI or to another instrument mounted on the port on the opposite side of the telescope. All these telescope sub-systems are controlled by a server program which will run only on a Windows PC. Tele-

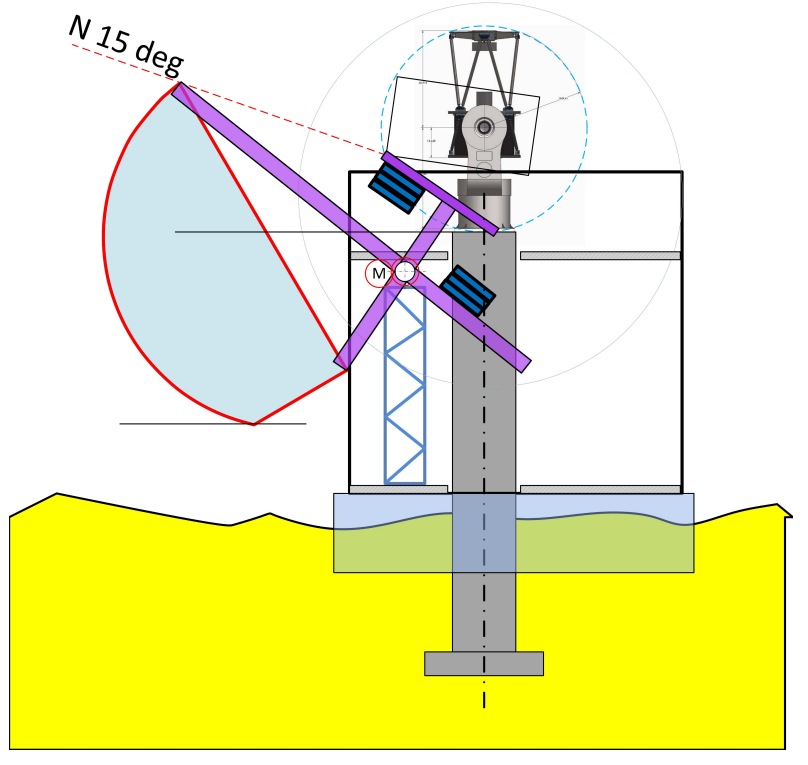

Fig. 4. Building and roof design.

scope command and communications are described below in Section $\S 3.1$.

\subsection{Cameras and BSTI Instrument}

In order to measure 2 independent spectral slopes of optical-IR emission a minimum of 3 spectral channels are required (Grossan et al. 2019). Figure 5 shows the design of the BSTI instrument indicating the 3 EMCCD cameras, and focus motors. These are mounted on a base plate mechanically coupled to the telescope at the Integrated Rotator Focuser (IRF90). We use $\mathrm{NuVu} 1 \mathrm{Kx} 1 \mathrm{~K}$ EMCCD cameras. The base plate was fabricated from aircraft aluminum, for enhanced stiffness. One fold mirror, for one camera, allows the system to fit on the mount without additional optics. Relative focus is maintained via 3 motorized translation stages which move the cameras along their optical paths. The EMCCD cameras allow very short exposures (as short as $115 \mathrm{~ms}$ ) with negligible read noise compared to conventional CCDs. The light from the telescope goes through the center of the IRF90. The bluest light (covering the Sloan g' filter) reflects off dichroic splitter 1, then reflects from the mirror and enters EMCCD 1. The remaining light in the middle of our band, covering the Sloan r' filter band, reflects from beam splitter 2 and enters EMCCD 2. The reddest of the light entering the telescope, covering the Sloan i' band, reaches EMCCD 3.

\section{SOFTWARE}

The software system is based on the GNU data language (GDL) in a Linux environment, selected for 


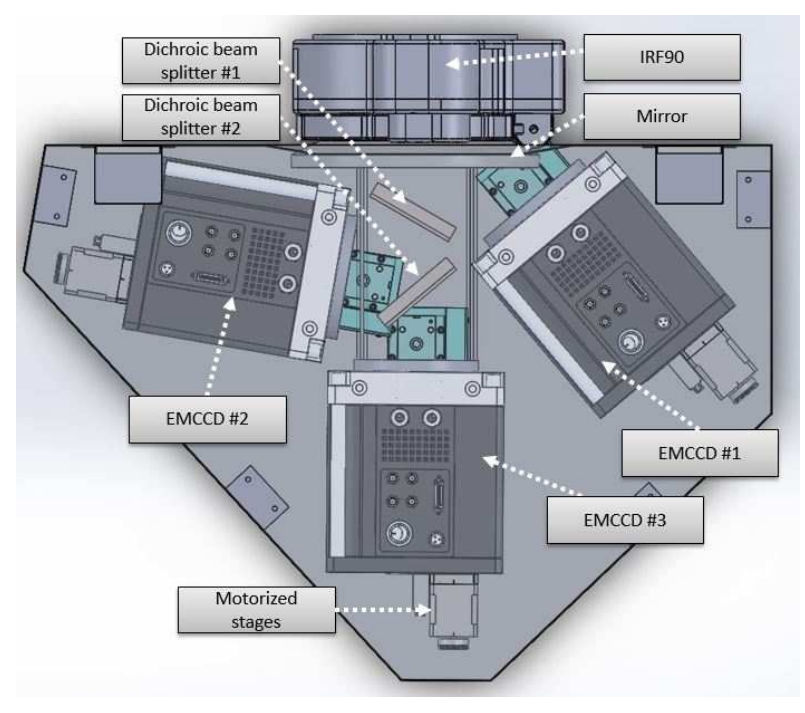

Fig. 5. BSTI design.

ease of writing and de-bugging software, familiarity to the project scientists, and image analysis capabilities. Linux environment is free licensed and provides stable performance in the long-term. Figure 6 shows software main scripts and subroutines in autonomous mode. In this mode, the observatory operates without human interaction. There are two independent control procedures. The Captain procedure, written in GDL, is responsible for all main process: alert listener, weather data check, telescope operations, camera operations and roof open/close. A separate control procedure is responsible for power source control. If the charge drops down to 40 percent, the procedure will activate the observatory diesel generator until the battery is fully charged.

\subsection{Hardware control}

In our solution we use devices with their own medium-to-high level commands as building blocks in the system. The captain procedure controls devices via commands to the Linux system, (the "spawn" command in GDL). Spawn allows execution of different commands asynchronously. In autonomous mode, the hardware equipment uses the following communication formats: Power: The ioLogik logic controller is used to control relays to power individual hardware components on and off, accepting HTTP requests in JSON format from the Captain procedure; Telescope mount: The telescope mount is controlled by high level HTTP requests. Mount sends back an xml formatted response with all parameters information; Telescope shutters: The telescope shutters works independently from the telescope mount, and are controlled by scripts written in Python. It has the following state commands:

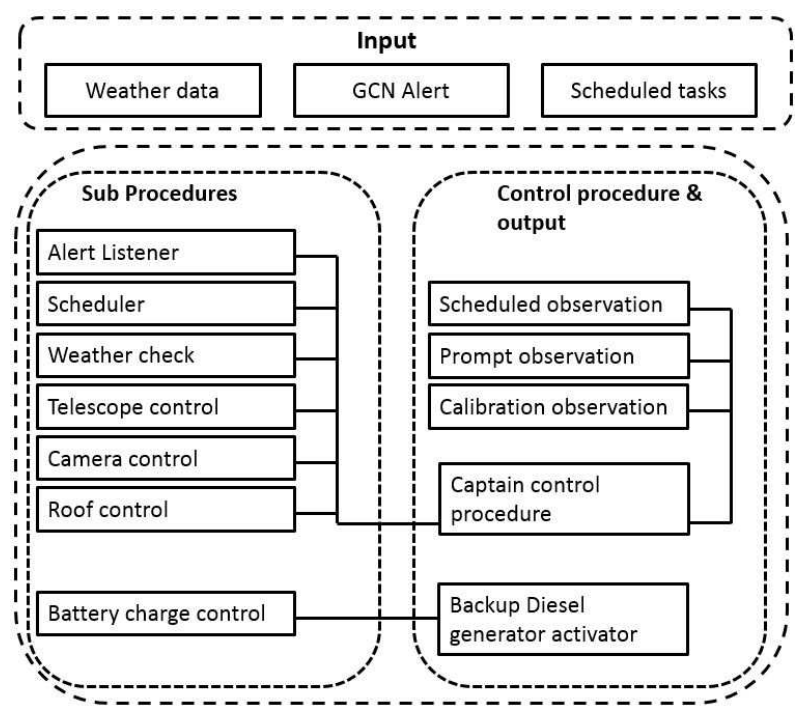

Fig. 6. Block diagram of software components in robotic mode.

connect, state status, begin open, and begin close; $E M C C D$ camera: EMCCD camera connected to IPC via GigE interface. Full camera control including cooler system, electron multiplication values, and different acquisition modes, operates via SDK functions (written in $\mathrm{C}$ ).

\subsection{Alert listener}

The 'alert listener' script receives real-time GRB position alerts via internet socket from the various instruments on the Gamma Coordinates Network (GCN) (Barthelmy et al. 1995). The Swift is most important for us, as it yields frequent GCN alerts with a position error smaller than our field. When 'alert listener' receives a socket alert, it sends the GRB position to the Captain procedure.

\subsection{Captain Procedure}

The Captain procedure is a GDL program that controls the observatory system during robotic mode. The main workflow of the script based on Finite State Machine model (FSM). FSM model has states and conditions. For NUTTelA-TAO we have built a model with cameras, roof and telescope as states; time, weather and alert as conditions (Figure 7). "Weather False" and "Weather True" means inappropriate and appropriate weather condition for observations, respectively. "Time True" condition works during night time, immediately after sunset, and immediately before sunrise. During the day time (Time False), the system remains in "Idle" state, when equipment is turned off. "Alert True" condition requires alert packet from GCN socket. "Roof 
TABLE 1

RESPONSE TIME IN AUTONOMOUS MODE

\begin{tabular}{lcc}
\hline Name of the process & Duration of process (seconds) Total time (seconds) \\
\hline Read socket alert and calculate availability inside FOV & 0.003 & 0.003 \\
Target telescope (maximum slew time) & 7.892 & 7.895 \\
Trigger camera 1 & 0.009 & 7.904 \\
Trigger camera 2 & 0.021 & 7.925 \\
Trigger camera 3 & 0.007 & 7.932 \\
Camera inner delay before image acquisition & 4.974 & 12.906 \\
\hline
\end{tabular}

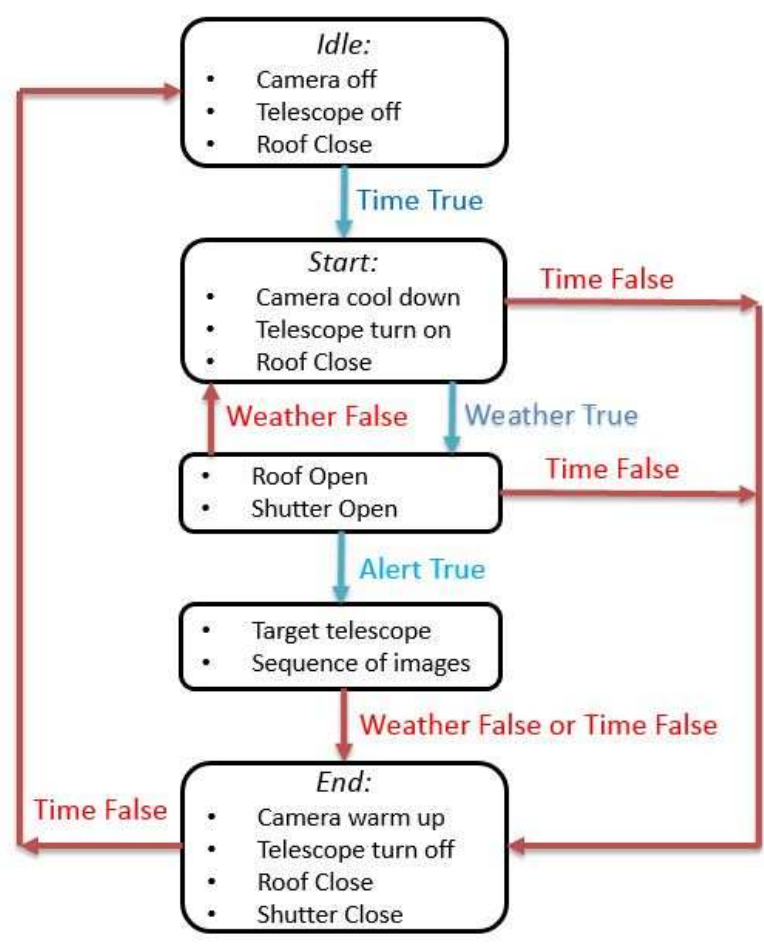

Fig. 7. State machine diagram for automation system.

Open" state means that the weather condition suitable for night observations. There is no risk of damaging hardware equipment.

\section{PERFORMANCE: RESPONSE TIME}

All the performance data given in this section refer to the final configuration of the telescope described in Sections 2 and 3. A big challenge for this project is to measure majority of the duration of the prompt optical emission of GRBs. It can take 20 or more seconds for the Swift satellite to detect and localize GRBs and transmit the position to the ground. The GCN distributes this position information within $3 \mathrm{~s}$. over the internet. We assume that NUTTelA-TAO will miss the beginning of the GRBs, but measure the majority of the bright prompt optical-IR emission. The log T90 (time for 90 percent of the burst fluence) duration distribution of Swift long GRBs peaks at around $70 \mathrm{~s}$ (Sakamoto et al. 2011). A short response time is crucial to cover more than a half of the long GRBs. To estimate total response time we assume that the weather condition is suitable for observations, the roof is open, the cameras are cold, the telescope is at home position and it has to move to the opposite side of the sky (largest possible angular distance). Values presented in Table 1 are the average numbers of 30 runs. The total response time is 13 seconds in average.

\section{CONCLUSION}

We have accomplished full observatory automation, including telescope, roof and instrument with short response time. With 13 seconds response time, GRBs in the field of view, and appropriate weather condition at the right time, NUTTelA-TAO is capable to measure the majority of the duration of prompt optical emission of GRBs. Our unusual solution with multiple communication protocols and programming languages shows reliability in the long term test. With automated alert response, the next step will be long GRBs observations and measurements of optical spectral shape during the prompt phase.

\section{REFERENCES}

Barthelmy, S. D., Butterworth, P., Cline, T. L., et al. 1995, Ap\&SS, 231, 235

Grossan, B., Kumar, P., Smoot, G.F. 2019, JHEAp, 23, 14

Li, S., Smoot, G. F., Grossan, B., et al. 2019, arXiv eprints, arXiv:1908.10549

Marchant, J., Smith, R. J., Steele, I. A. 2008, Proc. SPIE, $70123 \mathrm{U}$

Sakamoto, T., Barthelmy, S. D., Baumgartner, W. H., et al. 2011, ApJS, 195, 2

Shafiee M., Grossan B., Hu J. Colantoni I. Smoot G. 2019, JInst, 14, 2019 